\section{Synthese tetrasubstituierter Ethylene durch reduktive Kupplung von Ketonen mittels Titan(II)-salzen. Zur Anwendung der Methode}

Dieter LENOIR

Organisch-Chemisches Institut der Technischen Universität München, Arcisstraße 21, D-8000 München 2

Nachdem kürzlich die durch Titan(lI)-salze bewirkte reduktive Kupplung von Aldehyden und Ketonen von zwei Arbeitsgruppen beschrieben wurde ${ }^{1}$, ist diese Reaktion mehrfach zur Synthese von Pinakolen und Alkenen angewendet worden $^{2}$. Veranlaßt durch unser Interesse an Nachweis und Eigenschaften sterisch gehinderter Olefine ${ }^{3}$ haben wir die Möglichkeit untersucht, mittels dieser Methode durch sperrige Alkyl-Gruppen substituierte Ethylene herzustellen ${ }^{4}$. Die Methode wurde von uns modifiziert (Verwendung von $\mathrm{TiCl}_{4} / \mathrm{Zn}$ in Gegenwart von Pyridin) und ihre Anwendbarkeit an acht repräsentativen Ketonen aus der offenkettig-aliphatischen und cycloaliphatischen Reihe untersucht.<smiles>[R]C([R])=[O+]C([R])[R]</smiles>

1

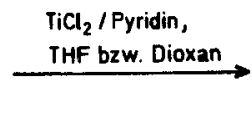<smiles>[R]C([R])=C([R1])[R]</smiles>

2
Die hier beschriebene Reaktion stellt eine präparativ brauchbare Methode zur Synthese symmetrisch alkyl- bzw. cycloalkyl-substituierter Ethylene dar. Die bisher berichteten Ver- fahren zur Herstellung der Alkene $2 d^{5}, 2 e^{6}$ und $2 f^{7}$ sind wesentlich aufwendiger und können wegen der guten $\mathrm{Zu}$ gänglichkeit der Reagenzien bei der Titan(II)-Methode mit dieser nicht konkurrieren. Die Synthese der noch nicht beschriebenen Alkene 2a, b, c, g, h zeigt deutlich die allgemeine Anwendbarkeit der Methode.

Die sogenannte McMurry-Methode [Ti(II)-salz aus $\mathrm{TiCl}_{3} /$ $\left.\mathrm{LiAlH}_{4}\right]^{2}$ ergibt nach unserer Erfahrung keine wesentlich höheren Ausbeuten an Alken. Das von uns verwendete, im Vergleich zu Titan(III)-chlorid preiswertere Titan(IV)-chlorid hat außerdem den Vorteil, daß es sich von den Produkten seiner partiellen Hydrolyse durch Destillation leicht reinigen läßt. Die Reaktion führt nur dann zu optimalen Ausbeuten, wenn das Titan-salz absolut wasserfrei ist.

Die relativ niedrige Ausbeute an den Alkenen $2 c$, e, f, g, h wird durch die konkurrierende Bildung von sekundärem Alkohol verursacht, der sich bei der Chromatographie an Kieselgel mit Pentan/Ether $(4+1)$ eluieren und isolieren läßt. Bei sterisch zu stark gehinderten Ketonen wie Di-t-butyl-keton kann mittels der Titan(II)-Methode keine reduktive C-C-Kupplung erreichen; man beobachtet ausschließlich Reduktion der Carbonyl-Gruppe zur sekundären alkoholischen Gruppe 4 .

Die Stereoselektivität der Reaktion ist nicht sehr ausgeprägt, falls sich die entstehenden cis- und trans-Alkene in ihrem Energie-Inhalt nicht hinreichend unterscheiden. Für die unsymmetrischen Alkene 2a, $\mathbf{2 b}$ und $\mathbf{2} \mathbf{g}$ findet man laut gaschromatographischer Analyse ein Isomeren-Verhältnis von $\sim 1: 3,1: 4$, bzw. 1:3 (die Hauptmenge ist jeweils wahr-

Tabelle 1. Tetrasubstituierte Ethylene (2) aus Ketonen (1) mittels Titan(IV)-chlorid/Zink/Pyridin

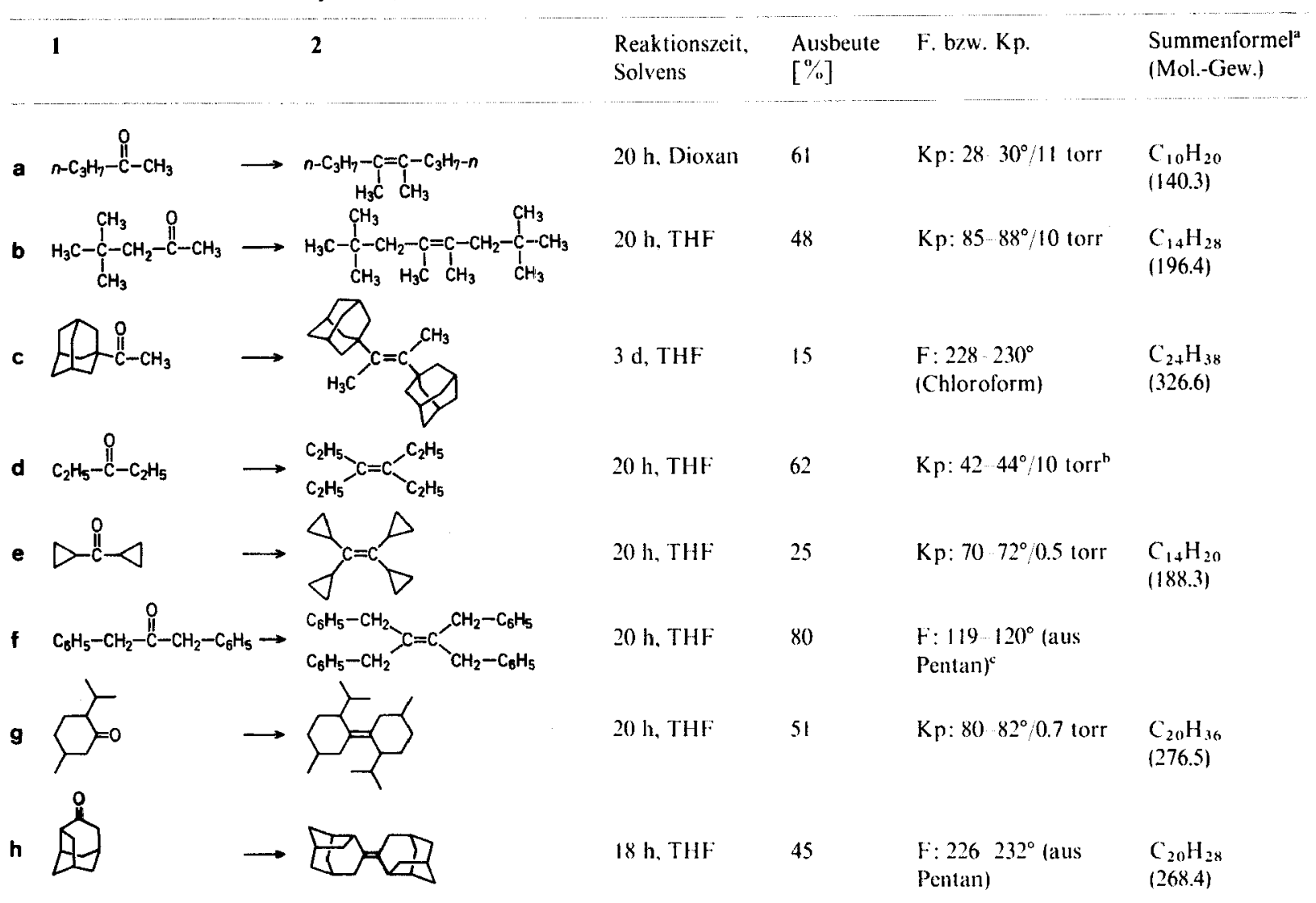

Die Mikroanalysen der neuen Verbindungen zeigten die folgenden maximalen Fehler: $\mathrm{C}, \pm 0.08 \% ; \mathrm{H} . \pm 0.04 \%$. Für dic Analysen wurden die flüssigen Produkte von Natrium abdestilliert.
${ }^{b}$ Lit. $.^{5}, \mathrm{Kp}: 158.1^{\circ} / 758$ torr.

' Lit. ${ }^{7}, F: 119.5-120.5^{\circ}$. 
Tabelle 2. Spektrale Daten der Alkene 2

\begin{tabular}{|c|c|c|c|}
\hline 2 & $\begin{array}{l}\text { M.S. }(70 \mathrm{eV}) \\
m / e(\text { rel. Intensität })\end{array}$ & $\begin{array}{l}\text { I.R. (Film bzw. } \mathrm{KBr}) \\
v_{\max }\left[\mathrm{cm}^{-1}\right]\end{array}$ & $\begin{array}{l}{ }^{1} \mathrm{H}-\mathrm{N} . \mathrm{M} . \mathrm{R} .\left(\mathrm{CCl}_{3} / \mathrm{TMS}\right) \\
\delta[\mathrm{ppm}]\end{array}$ \\
\hline $\mathbf{a}$ & $\begin{array}{l}140(81), 112(6), 111(7), 89(75), 97(98), 83(18) \\
79(6), 77(6), 70(55), 69(100), 67(20), 57(14), 56 \\
(16), 55(100), 53(17), 43(31), 41(97)\end{array}$ & $\begin{array}{l}2960,2935,2875,1465 \\
1455,1378,1155,1090 \\
740\end{array}$ & $\begin{array}{l}0.89(\mathrm{t}, J=7 \mathrm{~Hz}) ; 1.33(\mathrm{~m}) \\
1.65(\mathrm{~s}) ; 2.03(\mathrm{t}, J=7 \mathrm{~Hz})\end{array}$ \\
\hline b & $\begin{array}{l}196(19), 140(20), 123(10), 121(8), 85(7), 84(100), \\
83(97), 81(9), 69(10), 67(7), 57(15), 55(7), 41 \\
(21)\end{array}$ & $\begin{array}{l}2960,2910,2875,1480 \\
1460,1395,1380,1368 \\
1240,1205\end{array}$ & $\begin{array}{l}0.92 \text { (cis), } 0.92 \text { (trans); } \\
1.68 \text { (cis), } 1.73 \text { (trans); } \\
2.06 \text { (cis und trans) }\end{array}$ \\
\hline c & $\begin{array}{l}324(10), 264(9), 219(55), 181(5), 169(10), 150(5) \\
136(13), 135(94), 131(77), 119(30), 114(10), 107 \\
(8), 100(42), 93(23), 79(20), 69(100), 55(14), 41 \\
(19)\end{array}$ & $\begin{array}{l}3060,2900,2840,1440 \\
1340,1095,1070,1020 \\
810\end{array}$ & 1.7, 1.93 (breite Signale) \\
\hline d & $\begin{array}{l}140(18), 111(75), 83(12), 69(100), 55(100), 43(6), \\
41(24), 39(6)\end{array}$ & $\begin{array}{l}2950,2930,2860,1455 \\
1370,1270,1145,1070 \\
1050,1030,910,855 \\
788,768\end{array}$ & $\begin{array}{l}0.96(1, J=7 \mathrm{~Hz}) ; 2.03(\mathrm{q} \\
J=7 \mathrm{~Hz})\end{array}$ \\
\hline e & $\begin{array}{l}190(3), 188(3), 160(21), 147(18), 145(27), 134(39), \\
133(12), 131(28), 119(38), 117(40), 115(16), 105 \\
(47), 103(10), 95(11), 93(22), 92(14), 91(100), 81 \\
(40), 79(61), 77(31), 67(29), 65(19), 55(23), 53 \\
(21), 51(16), 44(15), 43(11), 41(67), 39(47)\end{array}$ & $\begin{array}{l}3080^{\mathrm{a}}, 3000,2960,2925 \\
2870,1455,1435,1375 \\
1255,1170,1100,1045 \\
1018^{\mathrm{a}}, 970,900,820\end{array}$ & $\begin{array}{l}0.400 .75(\mathrm{~m}, 12 \mathrm{H}) \\
0.93,1.35(\mathrm{~m}, 4 \mathrm{H}, J==7 \mathrm{~Hz})\end{array}$ \\
\hline f & $\begin{array}{l}389(19), 388(55), 297(44), 220(13), 218(10), 208 \\
(22), 207(100), 206(15), 193(31), 181(32), 179(12), \\
178(25), 167(25), 141(26), 129(55), 128(16), 117 \\
(61), 116(23), 115(47), 92(82), 77(12), 65(25)\end{array}$ & $\begin{array}{l}3075,3050,3020,2910 \\
2850,1595,1485,1445 \\
1245,1070,1025,905 \\
745,700\end{array}$ & $\begin{array}{l}3.52\left(\mathrm{~s}, 2 \mathrm{H}, \mathrm{CH}_{2}\right) ; 7.25 \\
\left(\mathrm{~s}, \mathrm{H}_{\mathrm{arom}}\right)\end{array}$ \\
\hline $\mathbf{g}$ & $\begin{array}{l}276(25), 274(6), 235(21), 234(100), 232(19), 177 \\
(41), 163(35), 149(15), 137(55), 135(12), 124(16), \\
123(98), 121(13), 109(91), 107(15), 105(20), 97 \\
(20), 95(86), 93(26), 91(27), 83(17), 81(51), 79 \\
(13), 69(30), 67(16), 55(27), 43(17), 41(26)\end{array}$ & $\begin{array}{l}2950,2920,2860,1450 \\
1380,1365,1220,1160 \\
1090,1020,970\end{array}$ & $\begin{array}{l}1.1,2.7,0.93(\mathrm{~d}, \text { breit, } \\
\left.\mathrm{CH}_{3}, J=7 \mathrm{~Hz}\right)\end{array}$ \\
\hline h & & $\begin{array}{l}2910,2850,1450,1350 \\
1330,1250,1095,1065 \\
975,800\end{array}$ & $\begin{array}{l}1.2,2.4 \text { (breite Signal- } \\
\text { gruppen) }\end{array}$ \\
\hline
\end{tabular}

${ }^{a}$ Lit, ${ }^{6} ; 3080$ bzw. $1020 \mathrm{~cm}^{-1}$

scheinlich das stabilere trans-Isomere). Das Produkt $\mathbf{2 h}$ ist aufgrund seines ${ }^{13}$ C-N.M.R.-Spektrums ein Gemisch von mindestens zwei Isomeren. Das Alken $\mathbf{2} \mathrm{c}$ ist stereochemisch einheitlich; ähnlich wic bei dem analog substituierten, durch reduktive Kupplung von $t$-Butyl-methyl-keton erhaltenen 2,2,3,4,5,5-Hexamethyl-3-hexen ${ }^{4}$ ordnen wir aus energetischen Gründen der Verbindung $2 \mathrm{c}$ die trans-Konfiguration zu.

Reduktive Kupplung von Ketonen zu Alkenen; allgemeine Arbeitsvorschrift:

Unter Rühren und Eiskühlung läß man im Stickstoff-Strom reines Titan(IV)-chlorid ( $14.2 \mathrm{~g}, 8.2 \mathrm{ml}, 75 \mathrm{mmol}$ ) zu absolutem Tetrahydrofuran oder Dioxan $(200 \mathrm{ml})$ tropfen. Anschließend gibt man Zinkstaub $(10 \mathrm{~g}, 150 \mathrm{mg}$-atom) in kleinen Portionen $\mathrm{zal}$, welcher sich infolge Bildung von Titan(II)-salz schwarz färbt. Dann wird Pyridin $(5 \mathrm{ml})$ zugegeben und danach eine Lösung des Ketons $(70 \mathrm{mmol})$ in Tetrahydrofuran bzw. Dioxan $(20 \mathrm{ml})$. Das Gemisch wird unter Rühren am Rückfluß erhitzt (Zeit siehe Tabelle 1). Nach dem Abkühlen läßt man zu der Suspension eine 10\%ige Lösung von Kaliumcarbona! $(150 \mathrm{ml}$ ! tropfen. Das Genisch wird mit Ether oder Pentan extrahiert $(5 \times 50 \mathrm{ml})$. Der Extrakt wird zweimal mit Wasser gewaschen, mit Magnesiumsulfat getrocknet, filtriert und im Vakuum eingedampft. Der Rückstand wird an Kieselgel (Merck 60, 0.2-0.6 mm: $60 \mathrm{~g}$ ) mit Pentan chromatographicrt. Das Alken befindet sich in der 1. Fraktion $(150 \mathrm{ml})$. Es wird zur weiteren Reinigung umkristallisiert $(2 \mathbf{c}$ aus Chloroform, $\mathbf{2 f}$ und $\mathbf{2 h}$ aus Pentan) oder destilliert.
Hern R. Frank und Frl. H. Müller sei für experimentelle Mitarbeit gedankt.

Eingang: 2. März 1977

' S. Tyrlik, 1. Wolochowicz, Bull. Soc. Chim. Fr. 1973, 2147. T. Mukaiyama, T. Sato, J. Hanna, Chem. Lett. 1973, 1041.

2 J. E. McMurry, M. P. Fleming. J. Am. Chem. Soc. 96, 4708 (1974)

R. F. Langler, T. T. Tidwell, Tetrahedron Lett. 1975, 777.

D. S. Bomse, T. H. Morton, Tetrahedron Lett. 1975, 781.

H. Wynberg, K. Lammertsma, L. A. Hulshoff, Tetrahedron Lett. 1975,3749

G. A. Olah, G. K. Surya Prakash, G. Liang, Symthesis 1976 , 318.

E. J. Corey. R. L. Danheiser, S. Chandrasekaran, J. Org. Chem. 41. 896 (1976).

A. J. Baumstark, E. J. H. Bechera, M. J. Semigran, Tetrahedron Let. 1976, 3265 .

P. D. Mollere, K. N. Hauk, D. S. Bomse, T. H. Morton, J. Am. Chem. Soc. 98,4732 (1976).

3 D. Lenoir, Tetrahedron Lett. 1972, 4049

D. I enoir, J. Firl, Justus Liehigs Ann. Chem. 1974, 1467.

4 D. Lenoir, $O$. Ermer, Veröffentlichung in Vorbereitung.

" H. Koch, F. Hilberath, Ber. Dtsch. Chem. Ges. 73, 1171 (1940).

6 A. Nierth, H. M. Ensslin, M. Hanack, Justus Liehigs Ann Chem. 733, 187 (1970).

H. A. Staab, H. A. Kurmeier, Chem. Ber, 101, 2697 (1968). 